\title{
EMULSION (OIL-IN-WATER) FLUID FLOW THROUGH CURVED DIFFUSER
}

\author{
K. A. Ibrahim ${ }^{1}$, W. A. El-Askary ${ }^{1}$, E. Wahba ${ }^{1}$ and Hamdy A. Omara ${ }^{2}$ \\ 1 Mechanical Power Engineering Department, Faculty of Engineering \\ Minoufiya University, Shebin El-Kom, Egypt \\ 2 Advanced Industrial Technical Arish School, North Sinai
}

\begin{abstract}
The paper presents a comprehensive experimental study on emulsion (oil-in-water) fluid flow through curved diffusers. The experimental setup was designed and constructed in the fluid mechanics laboratory of the faculty of engineering, Minoufiya University to perform the measurements which have been carried out on five models of curved diffusers. The measurements of pressure distributions along the outer and inner walls of the curved diffuser were performed for different area ratios, different curvature ratios (ratio of centerline arc radius to inlet width), different inflow Reynolds numbers and different emulsion holdup (ratio of oil volume to emulsion volume), while the oil concentration was varied from $0 \%$ to $20 \%$ by volume. The experimental work was carried out using two sets of oil-in-water emulsions; the first is stabilized o/w emulsion using Sodium Dodecyl Sulfate (SDS) emulsifier and the second is unstable (oil-in-water) emulsion at different holdup values. The energy-loss coefficients for each model are based on detailed measurements of the wall pressure distributions along walls of the curved-diffuser models including long upstream and downstream tangents. The energy-loss coefficient data were plotted as a function of Reynolds number for the tested models at different concentrations.

New results on energy-loss coefficient during flow of destabilized and stabilized oil-in-water emulsions through curved diffusers are reported in the present paper. The diffuser energy-loss coefficient is strongly affected by the geometrical parameters of diffuser, Reynolds number and emulsion holdup. Generally for the flow in curved diffusers, the resistance coefficients for stable and unstable (oil-in-water) emulsions were higher than that of pure-water flow. It is also noticed that the unstable oil-in-water emulsion exhibits lower values in loss coefficient compared with that given for stable (oil-in-water) emulsion.

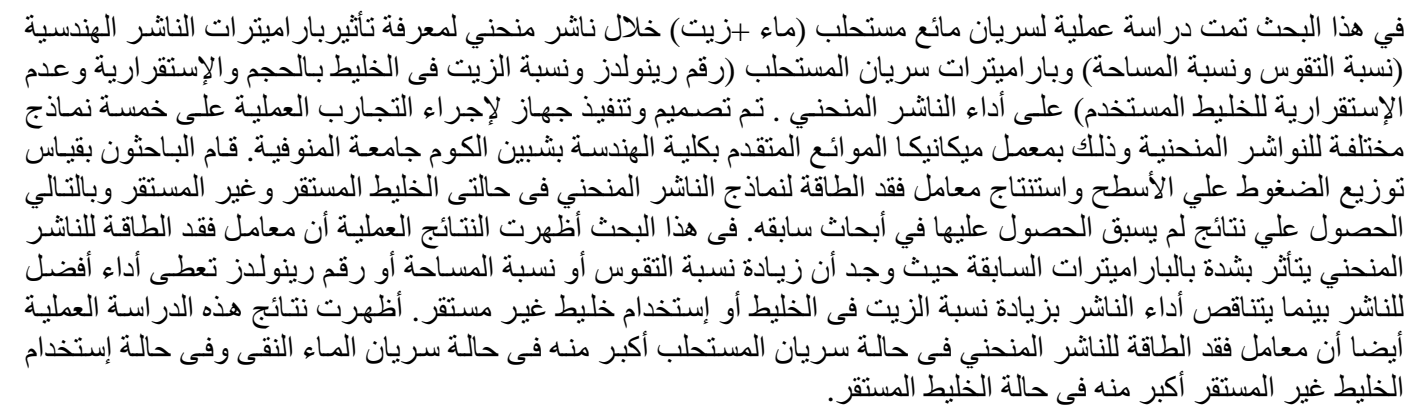

Keywords: Holdup, Emulsion flow, Stable, Unstable, Energy losses, Curved diffusers models

\section{INTRODUCTION}

The emulsion is a mixture of two immiscible liquids, one of which is dispersed in the form of small droplets throughout the other. The dispersed liquid is known as the internal or discontinuous phase whereas the dispersion medium is known as the external or continuous phase. As an important application, the two-phase (oil/water) emulsion flow is used for extraction of the petroleum oil from under ground by injecting the oil by water. Emulsions are also used in 
many other industries, such as pharmaceutical, agriculture and food industries. Curved diffusers are necessary in fluid flow to decelerate and turn the fluid simultaneously. Curved Diffusers are used in vaned diffusers of centrifugal pump passages, in steam turbine and in the interconnecting ducting between the components of the gas turbines. Literature review on single phase flow through curved diffuser indicates that the overall pressure recovery coefficient of the curved diffuser increases by increasing the inflow Reynolds number, by increasing the turbulence intensity and by increasing area ratio. Curved diffuser flow constitutes the most complex type of flows because they are rotational and three dimensional in shape for small aspect ratio. Consequently, curved diffuser flows are difficult to investigate both experimentally and numerically. Curved diffuser of varying lengths and aspect ratios has been employed in the past to study the streamline curvature effects and secondary motions in single phase flows. One of the main features of flow in curved diffuser is the presence of centrifugal force (because of the curvature) which gives rise to the radial pressure gradient normal to the streamline direction which varies inversely with the radius of curvature of the streamline and consequently pressure driven secondary motion. Curvature also affects turbulence structure (i.e., the Reynolds stresses) and hence the stress-driven secondary is much smaller and may not be observable.

A review of the literature indicates basically two types of experiments on single phase flow. In the first one, developing flow in curved diffusers of large aspect ratio has been measured to study the effects of convex or concave curvature on a nominally twodimensional turbulent boundary layer. The aspect ratio of curved diffusers is defined as the ratio of its cross-section height to the inlet width. The second type of experiments has been conducted mostly in curved diffuser with short or long straight sections upstream of the curved diffuser to study the evaluation of the secondary motion developing and fully developed flows.

Reneau et al. [1] studied the design and performance of curved diffusers. They concluded; that for single phase air flow and at the same flow Reynolds number, the pressure coefficient recovery of the curved diffuser is lower than that of straight diffuser and the curved diffuser efficiency is lower than the straight diffuser efficiency.

The mean flow field in a diffusing bend of 40 degrees was measured by McMillan [3]. He found that; as a result of adverse pressure gradient there are secondary flows causing a flow momentum boundary layer fluid on the inner wall (convex wall) and this causes an excess thickening of the inner wall boundary layer. Also McMillan [3] concluded that low-speed fluid in the boundary layer on the top and bottom walls is forced towards the convex wall by the pressure gradient and continuity of flow accelerates the fluid in the central portion of the channel towards the concave wall.

Sullerey [4] performed a comparison between straight diffuser and curved diffuser. This comparison was experimentally performed under the same area ratio, effective divergence angle and air flow Reynolds number. He concluded that the straight diffuser performance is bigger than that of curved diffuser. With increasing the free-stream turbulence the pressure recovery coefficient for each type increases.

The hydraulic resistances of emulsion (oil in water) flow across orifice-meter, venturi-meter, sudden contraction and sudden enlargement were measured by Nasr [5]. She did an experimental study to compare pressure drop across these geometries under different Reynolds number and different oil concentration by weight. The results showed that under the same flows Reynolds number; increasing the oil concentration causes increasing in the energy losses. Under the same oil concentration; increasing flow Reynolds number causes a decreasing in energy-loss coefficient.

Pal and Rhodes [6] investigated experimentally the laminar and turbulent flow behaviors of stable oil-inwater $(\mathrm{o} / \mathrm{w})$ emulsions in horizontal pipeline. They noticed that; up to a dispersed phase concentration of $55.14 \%$ by volume, emulsions are Newtonian. For concentration of $65.15 \%$ o/w emulsions are nonNewtonian power-law fluids. It was concluded that friction factor for stable o/w emulsions investigated follows the usual equations of single-phase Newtonian and non-Newtonian fluids with averaged properties. It was also pointed out that for dispersed phase concentration $>50 \%$ stable o/w emulsions exhibit drag reduction in turbulent flow, i.e.; the experimental friction factors fall somewhat below the single - phase equation.

An experimental study concerning the laminar and turbulent flow behaviors of unstable and stable water-in-oil (w/o) and oil-in-water (o/w) emulsions was performed by Pal [7]. The results showed that; the unstable o/w emulsions exhibit drag reduction behavior in the turbulent regime depending upon the nature of the oil and the holdup of the dispersed phase. The unstable w/o emulsions exhibit much stronger drag reduction activity than the unstable o/w emulsions. The stable emulsions exhibit relatively little drag reduction; therefore the pipeline flow behavior of stable o/w and w/o emulsions could be 
predicted reasonably well using the usual equations of single - phase flow.

Hwang and Pal [8] experimentally measured the flow of two-phase oil/water mixtures through sudden expansions and contractions. The energy losses were determined from the measured pressure profiles upstream and downstream. The loss coefficients for the emulsions were found to be independent on the type of emulsion. Also the results indicated that the emulsions are of oil-in-water type up to $64 \%$ (volume) oil and above this ratio the emulsions are water-in-oil type.

Turian et al. [9] found that the resistance coefficients for non-Newtonian suspension flow through bends of various angles and radii of curvature and through valves decrease with increasing Reynolds number in laminar flow. However, constant asymptotic values of resistance are approached in fully developed turbulent flow.

Pal and Hwang [10] presented an experimental study concerning the loss coefficients for flow of surfactant-stabilized emulsions through pipe components (valve, expansion and contraction). They showed that emulsions are Newtonian only at low to moderate concentrations of dispersed phase (oil concentration up to 46.9\%). Also, they concluded that the frictional losses can be successfully correlated as loss coefficient versus Reynolds Number.

Khalil et al. [11] performed an experimental study for flow of water with surfactant additives through sudden enlargements, sudden contractions and short bends. The results showed that the reduction in secondary losses increases by increasing of both surfactant concentration and Reynolds number and/or decreasing pipe diameter.

Langevin et al. [12] found that dispersing heavy oil or bitumen in water is a very efficient way to reduce the viscosity of the fluid by more than 2 orders of magnitude. Thus, formation of oil-in-water emulsions can be used to reduce the viscosity in pipelines. Therefore reducing energy losses and the required power to transmit the oil in pipelines.

Ismail [13] studied theoretically the drag reduction behavior of the unstable w/o emulsions in turbulent flow in a horizontal pipeline. The drag reduction is considered to be due to two reasons, the damping of turbulence due to the presence of dispersed phase, and due to breakage and coalescence processes. Thus, two correlations were obtained to describe the function in saucer mean diameter, holdup and diameter. The correlations were found to fit in good agreement with the experimental results of Pal [7].

A study of oil-in-water emulsion flow through pipeline using image analysis technique was performed by Khalil et al. [14]. The emulsion was prepared by adding oil to tap water and the results indicated that: Increasing emulsion holdup (ratio of oil volume to mixture volume) causes increasing in emulsion viscosity, increasing oil droplet diameter and decreasing discharge coefficient and also increasing energy-losses coefficient. Energy-loss coefficients of $90 \mathrm{o}$ bend, fully opened gate valve, sudden enlargement and sudden contraction were arranged according to the big value respectively. The resistance coefficient of pipe fittings for emulsion flow is lower than that for water flow. It was concluded that; for holdup lower than $50 \%$ the o/w emulsion flow is Newtonian flow and for holdup higher than $50 \%$ the o/w emulsion flow is nonNewtonian flow.

The pressure drop in sudden contractions was measured for Newtonian and non-Newtonian fluid under different flow Reynolds number by Fester et al. [15]. The experimental study indicated that: Contraction Loss coefficient $\left(\mathrm{K}_{\text {con }}\right)$ decreases with increasing contraction ratio $\left(\mathrm{D}_{\mathrm{d}} / \mathrm{D}_{\mathrm{u}}=\right.$ Ratio of downstream pipe diameter to upstream pipe diameter). The study showed that increasing flow Reynolds number decreases contraction loss coefficient.

The turbulent flow inside a combined bend-diffuser configuration with a rectangular cross section was experimentally and numerically studied by El-Askary and Nasr [16]. The experimental study included the outer and inner-wall-pressure measurements and the overall system/diffuser loss determination. The results show that there is an optimum diffuser angle which depends on the inflow Reynolds number and produces the minimum pressure loss and hence good performance of such complex geometry is obtained.

The pressure drop of oil-water flow through sudden contraction and expansion in a horizontal pipe was measured by Balakhrisna et al. [17]. The abrupt change in area during oil-water flow through pipe strongly affects on the phase distribution. The loss coefficient is independent on the flow patterns. Contraction and expansion coefficients are found to be lower for oil-in-water flow in comparison to pure water flow though the same test rigs.

Hammoud et al. [18] studied the effect of oil-in-water concentration on the performance of a centrifugal pump. They concluded that the head and efficiency decrease by increasing the oil concentration. 
Increasing in oil concentration leads also to increasing the power consumption.

Khalil et al. [19] measured the energy Losses of oilin-water emulsions flow through pipe fittings using image processing. They found that the energy loss coefficient increases as the holdup increases and the flow rate decreases; also they showed that the energy loss coefficient is found to be inversely proportional to the generalized flow Reynolds number.

For the authors' knowledge, up till now no work has been reported or published in the field of emulsions flow through curved diffusers. The main objective of the present work is to gain insight into the curved diffuser performance with emulsion (oil-in-water) flow. The effects of the inflow Reynolds number and the geometrical parameters of the curved diffuser such as the curvature ratio and area ratio are considered. The oil concentration in water (holdup) is also included in order to extract general conclusions. First of all, outer and inner wall static pressure distributions are experimentally measured in flow of stable and unstable oil-in-water emulsions through curved diffusers at different values of holdup and flow Reynolds numbers. Second, studying the energy losses of stable and unstable oil-in-water emulsions flow through curved diffusers at different values of holdup and Reynolds numbers are also extracted.

\section{Analytical Study}

Emulsions can generally be treated as pseudohomogeneous fluids with averaged properties as the dispersed droplets of emulsions are small and are well distributed. Consequently, one can apply the single-phase flow equation to correlate the pressure loss data for emulsion flow through diffusers. It should be noted that emulsions are Newtonian only at low to moderate concentrations of dispersed phase (oil concentration up to 46.9\%), see Pal and Hwang [10].

Bernoulli's equation is valid along any streamline in any frictionless flow, and it can be modified to include the energy losses due to the presence of curved diffuser wall friction and the superimposed vortices produced by curvature or recirculation. Thus, for the $90^{\circ}$ curved diffuser, the modified Bernoulli's equation applied between the inlet and exit of the diffuser is written as:

$$
\begin{aligned}
& h_{\text {inlet }}+\frac{V_{\text {inlet }}^{2}}{2 \mathrm{~g}}=h_{\text {exit }}+\frac{V_{\text {exit }}^{2}}{2 \mathrm{~g}}+\mathrm{h}_{\text {loss }} \\
& h_{\text {loss }}=\left(h_{\text {inlet }}-h_{\text {exit }}\right)+\frac{\left(V_{\text {inlet }}^{2}-V_{\text {inlet }}^{2} / \mathrm{AR}^{2}\right)}{2 \mathrm{~g}}
\end{aligned}
$$

$$
h_{\text {loss }}=\frac{\left(P_{\text {inlet }}-P_{\text {exit }}\right)}{\rho_{E} \mathrm{~g}}+\frac{V_{\text {inlet }}^{2}\left(1-1 / \mathrm{AR}^{2}\right)}{2 g}
$$

With $h_{\text {inlet }}$ and $h_{\text {exit }}$ are the pressure heads at inlet and exit, respectively, while $V_{\text {inlet }}$ and $V_{\text {exit }}$ are the time-averaged mean velocities at inlet and exit, respectively and $\rho_{E}$ is the mixture (emulsion) density. The pressure coefficient is defined as:

$$
C_{p}=\frac{\left(P_{x}-P_{r e f}\right)}{0.5 \rho_{E} U_{r e f}^{2}}
$$

Where, $P_{x}$ is the local measured wall measured pressure and $P_{\text {ref is the reference pressure and }}$ $U_{\text {ref }}=V_{\text {inlet }}$ is the reference mean velocity.

The reference location is taken at a distance $20 \mathrm{~mm}$ upstream of the curved diffuser entrance.

The loss coefficient can then be determined from the following equation:

$$
\mathrm{K}_{d}=\left(C_{p \text { (inlet) }}-C_{p(\text { exit })}+1-\frac{1}{A R^{2}}\right)
$$

From which the loss head can be computed as:

$$
\mathrm{h}_{\text {loss }}=K_{d} \frac{\mathrm{U}_{\text {ref }}^{2}}{2 \mathrm{~g}}
$$

Where $C_{p \text { (inlet) }}$ and $C_{p \text { (exit) }}$ are the pressure coefficients at inlet and exit of the diffuser, respectively and $A R=A_{\text {exit }} / A_{\text {inlet }}$ is the diffuser area ratio. The area ratios for the considered geometries are: $A R=2$ for models $1,2,3$ and 4 , while $A R=1.5$ for model 5. Model 6 constitutes a smooth-curved duct of $A R=1$, see Table 1 .

\section{EXPERIMENTAL SETUP AND PROCEDURE}

\section{2-1 Apparatus}

Figure (1-a) shows the test rig that is designed to investigate the $\mathrm{o} / \mathrm{w}$ emulsion flow through curved diffusers of constant height ( $B$ ) and inlet width (W) of $80 \mathrm{~mm}$ and $20 \mathrm{~mm}$, respectively, but different center-line arc radius $\left(R_{c}\right)$ of 100, 150 and $250 \mathrm{~mm}$ and exit width $\left(W_{\text {exit }}\right)$ of 20, 30 and $40 \mathrm{~mm}$, see Fig. (1-b).

The emulsion is prepared in a large tank $\left(0.5 \mathrm{~m}^{3}\right.$ capacity) equipped with two high shear mixers. 
The emulsion from the preparation tank is circulated through the pipeline-test rig using a centrifugal pump. The temperature during all experiments is maintained constant at $27^{\circ} \mathrm{C}$ with temperature controller.

The emulsion flow rate is measured by a calibrated orifice meter and controlled by a gate valve placed after the pump.

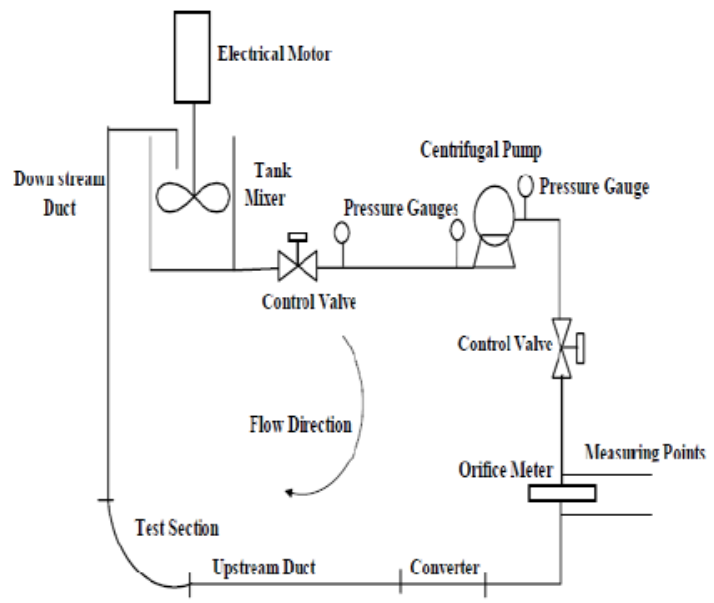

(a)

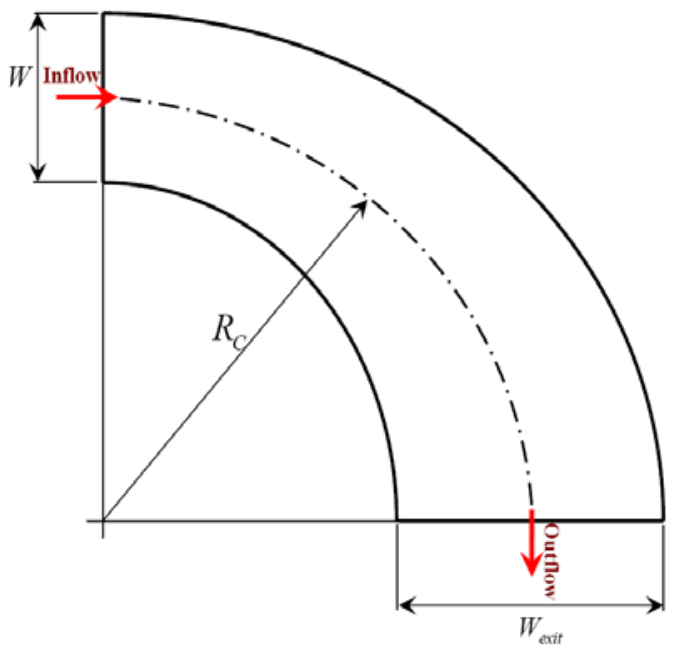

(b)

Fig.1 Schematic diagram of the experimental setup (a) and test section of the curved diffuser (b) (not to scale)

Figure (2) shows the experimental values of discharge coefficient with different emulsion holdup and different Reynolds number $\rho_{E} D_{p} \bar{V} / \mu_{E}$, where $\rho_{E}$ is the emulsion density, $D_{p}$ is the pipe diameter, $\bar{V}$ is the average emulsion velocity and
$\mu_{E}$ is the emulsion viscosity. The calibration curves of the orifice meter are clearly shown in Fig. (2).

Generally, as noticed the discharge coefficient of the orifice meter decreases with the holdup with very small variations with Reynolds number.

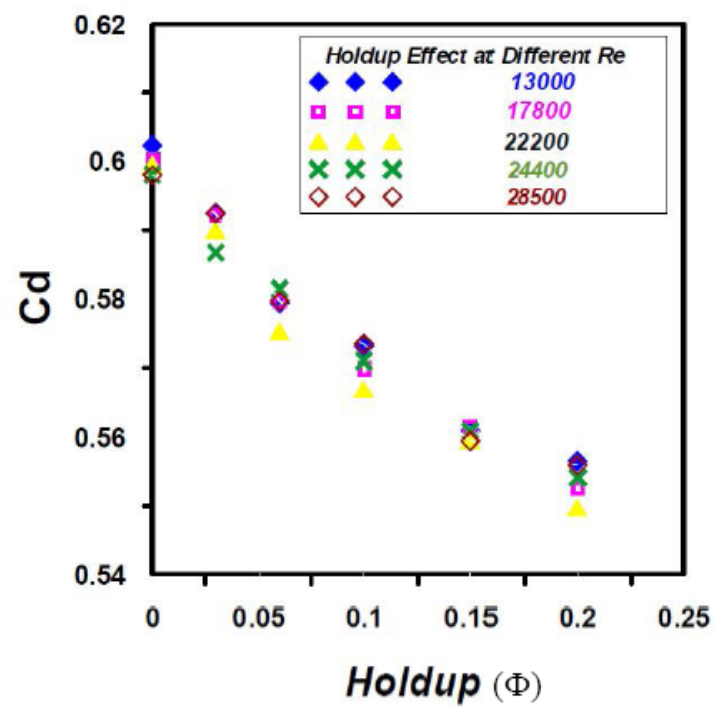

Fig. 2 Discharge coefficient of the orifice meter

\section{2-2 Emulsion Preparation}

Two different sets of emulsions are prepared using tap water and a refined white mineral oil. The white mineral oil is low viscosity colorless, tasteless and odorless highly refined paraffinic oil, supplied by CO-OP Company, Alexandria, Egypt. It has a density of $840 \mathrm{~kg} \mathrm{~m}^{-3}$ at $27^{\circ} \mathrm{C}$ and a kinematic viscosity limits at $40^{\circ} \mathrm{C}$ from 13 to $19 \mathrm{cSt}$. In the first set of emulsions, no chemical-emulsifier (surfactant) is added so that unstable emulsion is produced. The unstable emulsion will be separated into oil and water if left without agitation for sometime. The experiments in this set begin with tap water into which a required amount of oil varies from 0.0 to 0.2 (20\%) by volume based on the emulsion volume. The holdup $\Phi$ is defined as:

$$
\text { Holdup }=\Phi=\frac{V_{o}}{V_{o}+V_{w}}=\frac{V_{o}}{V_{E}}
$$

Where $V_{o}$ is the volume of oil, $V_{w}$ is the volume of water, and $V_{E}$ is the total (emulsion) volume. In the second set of emulsions an ionic surfactant (chemical-emulsifier) namely Sodium Dodecyl Sulfate $\left[\mathrm{CH}_{3}\left(\mathrm{CH}_{2}\right)_{11} \mathrm{OSO}_{3} \mathrm{Na}\right]$ is added to the oil with $1.5 \%$ based on the water weight.

The density and viscosity of pure water and oil-inwater emulsions are measured in laboratories of 
faculty of sciences, Minoufiya University using densitometer and viscometer. The measuring values of emulsions density and viscosity are summarized in a worksheet table by which the relation between the density as well as viscosity against the holdup for stable and unstable (oil-in-water) emulsions are drawn as shown in Fig. (3).

Figure 3 shows the relative density (emulsion density to water density) and relative viscosity (emulsion viscosity to water viscosity) at different holdup values of stable and unstable emulsions at the laboratory temperature $\left(27^{\circ} \mathrm{C}\right)$. As noticed, the density decreases while the viscosity increases as the holdup increases. The present measurements of the discharge coefficient, density and viscosity are considered for the sake of the exact computations of Reynolds numbers at inflow.

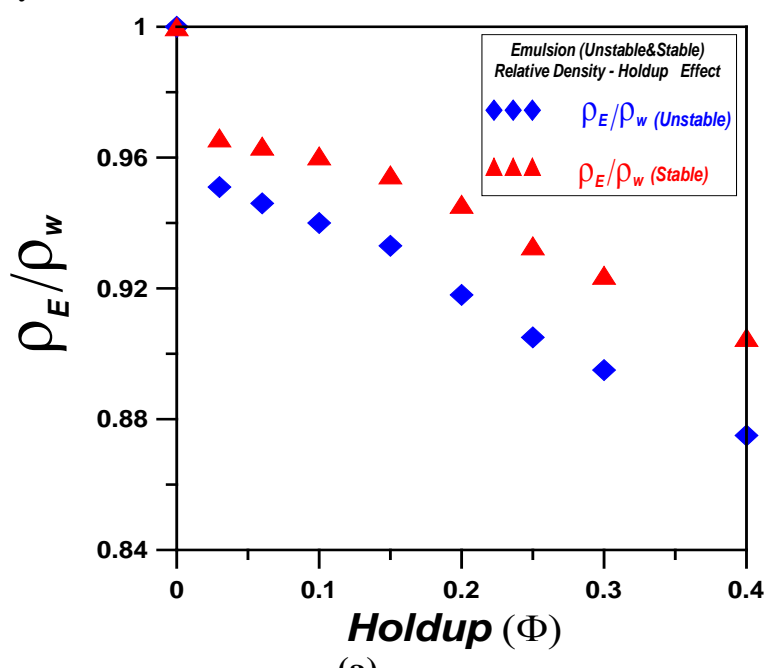

(a)

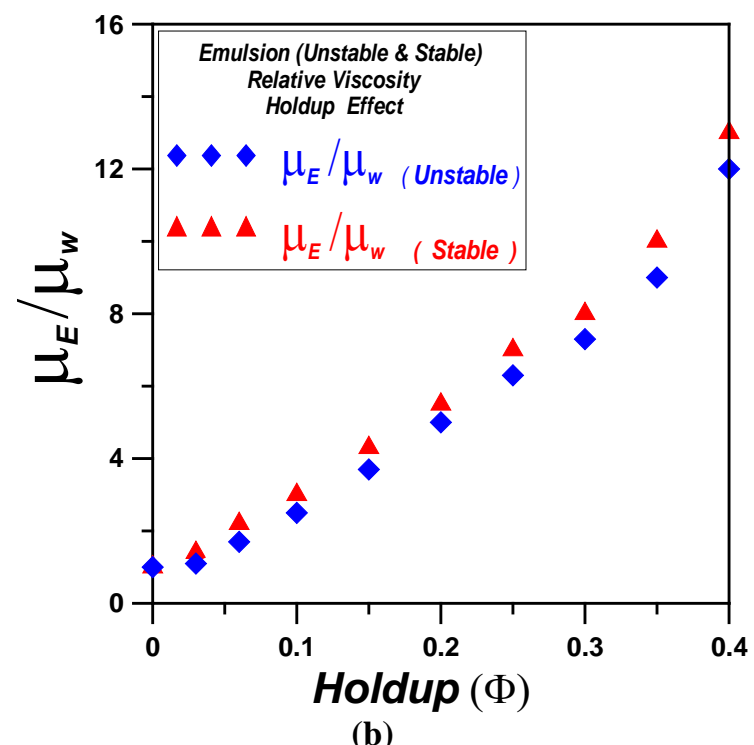

Fig. 3 Relative density (a) and viscosity (b) of unstable and stable oil-in-water emulsions versus holdup

\section{2-3 Wall Static Pressure Measurements}

Multi-tube manometer is used to measure the static pressure distributions along the outer and inner walls of the curved diffusers (test section), the upstream tangent and downstream tangent. The measuring fluid used in the manometer is Carbon Tetra Chloride $\left(C C L_{4}\right)$. This liquid does not mix with water as well as the emulsions and has specific gravity of 1.4 approximately. By using this liquid the static pressure at each tapping hole can be measured by the difference-head of the used measuring liquid.

\section{UNCERTAINTY ANALYSIS}

Before being documented for any analysis or design, the data obtained from experimental studies should be validated. The experimental errors may be of two types, namely fixed and random errors. Fixed error can be removed by proper calibration or correction while random error cannot be removed. The factors that introduce random error are uncertain by their nature. Uncertainty analysis should be conducted on all data collected from all measurements in order to quantify the data and validate the accuracy. As known, the flow resistance coefficient of the tested curved diffuser, $K_{d}$ (curved diffuser-energy loss coefficient) is given by:

$K_{d}=h_{\text {loss }} \frac{2 g}{U_{\text {ref }}^{2}}=h_{\text {loss }} \frac{2 g}{(Q / A)_{\text {ref }}^{2}}=h_{\text {loss }} \frac{2 g B^{2} W^{2}}{Q^{2}}$

In which, $Q$ is the volume flow rate measured by the calibrated orifice meter and $h_{\text {loss }}$ is the loss head through the curved diffuser. The precision uncertainty of $K_{d}$ is due to uncertainties for all independent variables of them. The precision Uncertainty $\left(\frac{\delta \mathrm{K}_{d}}{\mathrm{~K}_{d}}\right)$ can be represented as given in Taylor [2]:

$$
\frac{\delta \mathrm{K}_{d}}{\mathrm{~K}_{d}}= \pm\left\{\begin{array}{l}
\left(\frac{h_{\text {loss }}}{K_{d}} \frac{\partial K_{d}}{\partial h_{\text {loss }}} \frac{\delta h_{\text {loss }}}{h_{\text {loss }}}\right)^{2}+\left(\frac{B}{K_{d}} \frac{\partial K_{d}}{\partial B} \frac{\delta B}{B}\right)^{2} \\
+\left(\frac{W}{K_{d}} \frac{\partial K_{d}}{\partial W} \frac{\delta W}{W}\right)^{2}+\left(\frac{Q}{K_{d}} \frac{\partial K_{d}}{\partial Q} \frac{\delta Q}{Q}\right)^{2}
\end{array}\right\}^{1 / 2}
$$

where, $\quad \delta h_{\text {loss }} / h_{\text {loss }}, \delta B / B, \delta W / W \quad$ and $\partial Q / Q$ are the precision uncertainties for independent variables $h_{\text {loss }}, B, W$ and $Q$, respectively. The reasonable estimate of the uncertainty intervals due to random error in the present experimental study is taken as plus or minus half the smallest scale division (the least count) of the 
instrument used in conducting the measurement. Thus, $\delta h_{\text {loss }}, \delta B$ and $\delta W$ are taken to be $0.5 \mathrm{~mm}$. The discharge $\mathrm{Q}$ is measured using the calibrated orifice meter and calculated from the orifice meter equation as follows:

$$
Q=C_{d} \frac{A_{o} A_{p}}{\sqrt{A_{p}^{2}-A_{o}^{2}}} \sqrt{2 g \Delta H_{o} \frac{\rho_{m}-\rho_{E}}{\rho_{E}}}
$$

Where $C_{d}$ is the orifice discharge coefficient, $A_{o}=\frac{\pi}{4} d_{o}^{2}$ is the orifice area, $A_{p}=\frac{\pi}{4} D_{p}^{2}$ is the pipe cross-section area, $\Delta H_{o}$ is the mercury headdifference in the mercury U-tube manometer, $\rho_{m}$ is the mercury density in the manometer and $d_{o}$ is the orifice diameter. Thus,

$$
\frac{\delta Q}{Q}= \pm\left\{\begin{array}{l}
\left(\frac{\Delta H_{o}}{Q} \frac{\partial Q}{\partial \Delta H_{o}} \frac{\delta \Delta H_{o}}{\Delta H_{o}}\right)^{2} \\
+\left(\frac{D_{p}}{Q} \frac{\partial Q}{\partial D_{P}} \frac{\delta D_{P}}{D_{P}}\right)^{2} \\
+\left(\frac{d_{O}}{Q} \frac{\partial Q}{\partial d_{O}} \frac{\delta d_{O}}{d_{O}}\right)^{2}
\end{array}\right\}^{2 / 2}
$$

By substituting Eq. (11) into Eq. (9), the precision uncertainties of the energy-loss coefficient can be obtained.

Table (2) presents the range of energy loss coefficient and percentage precision uncertainty of resistance coefficient $\left( \pm U_{k_{d}} \%\right)$ during all measurements.

\section{RESULTS AND DISCUSSIONS}

\section{4-1 Wall Pressure Distributions}

In the present study, it is aimed to study the energyloss coefficients (resistance coefficients) for $90^{\circ}$ curved diffusers which have different curvature ratios $\left(C R=R_{c} / W\right): 5,7.5$, and 12.5, and different area ratios: $1,1.5$, and 2 . However, the geometrical parameters of all six models of the curved diffusers are given in Table 1.

The results are presented for unstable oil-in-water emulsions at different holdup $(\Phi)$ values from 0.03 to 0.06 and for stable oil-in-water emulsions at different holdup values from 0.03 to 0.20 . These measurements are performed at different Reynolds numbers range of $13,000 \leq \mathrm{Re} \leq 28,500$, where
$\operatorname{Re}=\frac{\rho_{E} U_{r e f} D_{H}}{\mu_{E}}$ with $D_{H}=\frac{2 W B}{W+B}$ (the hydraulic diameter). The pressure recovery coefficients are first presented to obtain the corresponding energy-loss coefficients of the curved diffusers with pure water as well as oil-in-water emulsion with the previous stated values of holdup for stable and unstable emulsions.

The static pressure recovery coefficient for all diffuser models are presented for pure water in Figs. (4) and (5) at water flow Reynolds number of 28,500. The effects of curvature ratio (Fig. 4) and area ratio (Fig. 5) are clearly shown, respectively

From the experimental results the comparisons in Figs. (4) and (5) reveal that the pure water flow in the curved diffuser model (3) exhibits the lower value in the static pressure recovery coefficient, then model (2) comes in the second stage, and the static pressure recovery coefficient in model (1) comes in the third stage. Also the comparison shows that the static pressure recovery coefficient of the curved diffusers with pure water increases as the area ratio or curvature ratio increases. This behavior tends to lower the resistance to the flow in case of high area ratio or curvature ratio. This same behavior is noticed with increasing the area ratio (models 4, 5 and 6) as shown in Fig. (5).

Generally the higher wall pressure distribution is observed on the outer walls of all models, because of the presence of radial pressure gradient generated by the centrifugal force.

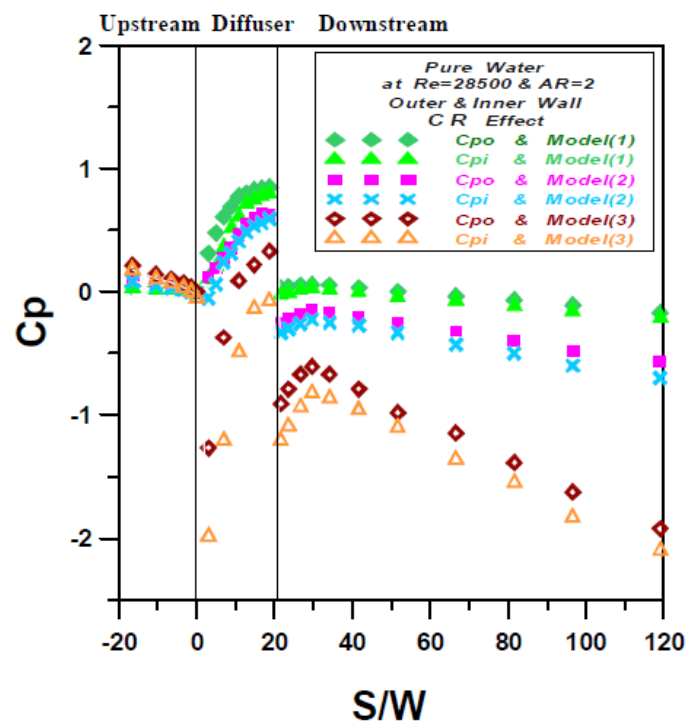

Fig. 4 Effect of curvature ratio on static pressure recovery coefficient on outer and inner walls for models (1), (2) and (3) with $\mathrm{AR}=2$ and $\operatorname{Re}=28,500$ (water flow) 


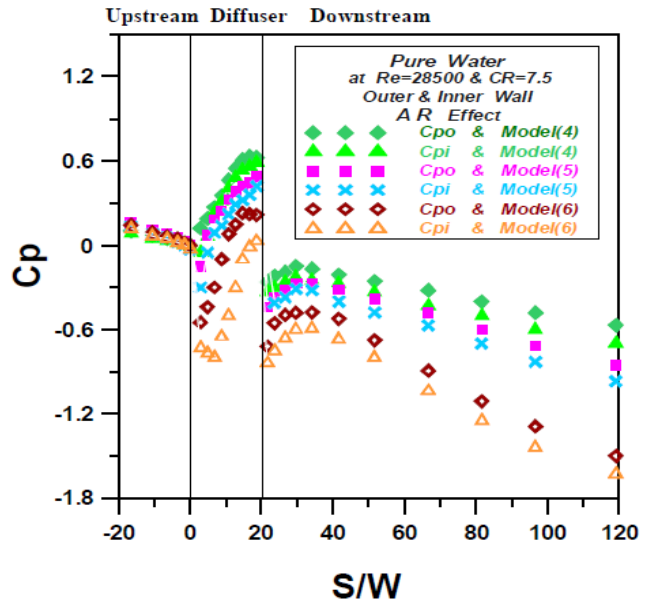

Fig. 5 Effect of area ratio on static pressure recovery coefficient on outer and inner walls for models (4),

(5) and (6) with $\mathrm{CR}=7.5$ and $\mathrm{Re}=28,500$ (water flow)

The static pressure recovery coefficients for all models are also presented for 0.15 stable oil-in-water emulsions in Figs. (6) and (7) on the outer and inner walls at emulsion flow Reynolds number of 28,500. By comparing the results in Figs. (6) and (7) it is found that the static pressure recovery coefficient increases as the area ratio or curvature ratio increases. This because as the area ratio, (AR) increases from 1.0 to 2.0, the emulsion flow resistance decreases and also the increase of curvature ratio from 5.0 to 12.5 leads to the emulsion flow resistance decreases. Comparing the presence of 0.15 oil concentration with case of water only, one notices that there is a distinct reduction of pressure along the walls of curved diffuser (compare Fig. (4) with Fig. (6) and Fig. (5) with Fig. (7).

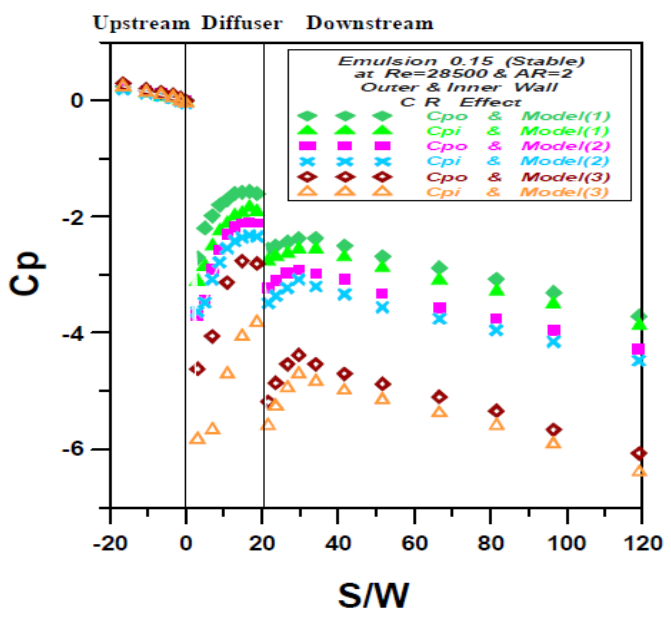

Fig. 6 Effect of curvature ratio on static pressure recovery coefficient on outer and inner walls for models (1), (2) and (3) with $\mathrm{AR}=2$ and $\mathrm{Re}=28,500$

(stabilized emulsion flow with $\Phi=0.15$ )

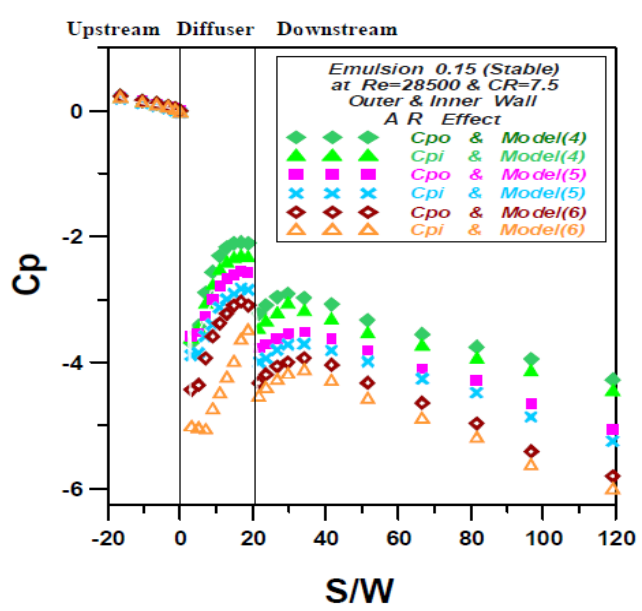

Fig. 7 Effect of area ratio on static pressure recovery coefficient on outer and inner walls for models (4), (5) and (6) with $\mathrm{CR}=7.5$ and $\mathrm{Re}=28,500$ (stabilized emulsion flow with $\Phi=0.15$ )



(a)

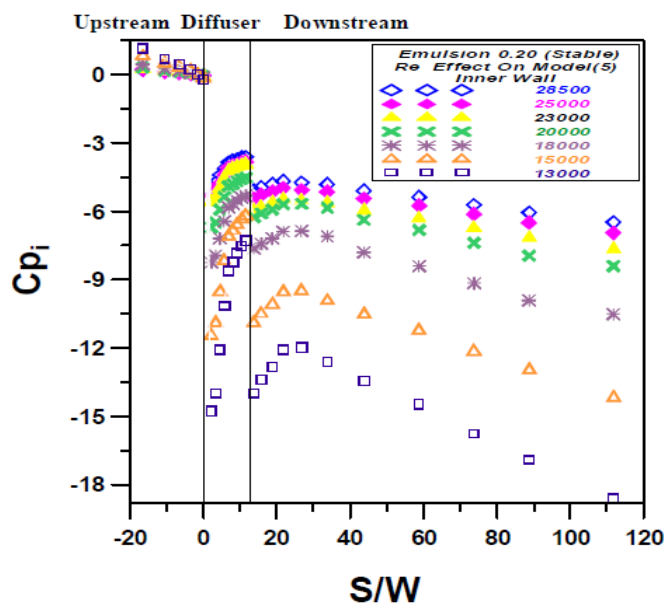

(b)

Fig. 8 Effect of Reynolds number on static pressure recovery coefficient on outer wall (a) and inner wall (b) for model (5) with $\mathrm{CR}=7.5$ (stabilized emulsion flow with $\Phi=0.20$ ) 
The static pressure recovery coefficient for model (5) is presented for a stable emulsion of 0.20 oil concentration in Fig. (8) on outer and inner walls at different emulsion flow Reynolds numbers, ranged

from 13,000 to 28,500 . It is noticed that the wallpressure recovery coefficient of the curved diffusers with 0.20 stable oil-in-water emulsions is directly proportional to the flow Reynolds number with a noticeable decrease in the adverse pressure gradient inside the diffuser. This leads to a decrease of the boundary layer thickness on the walls and hence reduces the chances of separation in the curved diffuser. Therefore, the performance of curved diffuser can be improved with accelerating the inflow.

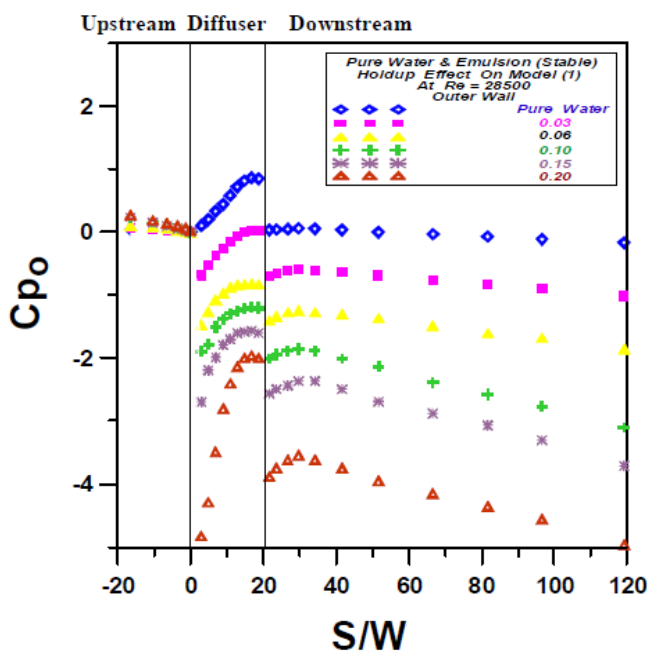

(a)

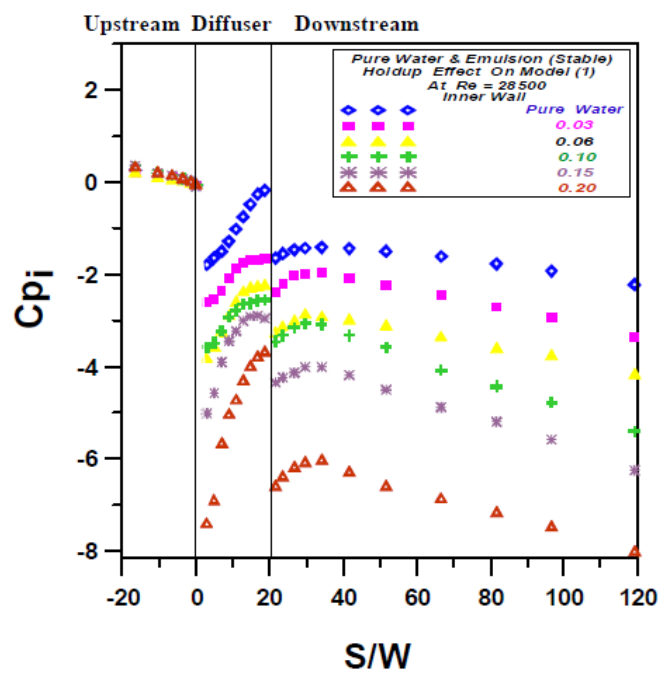

(b)

Fig. 9 Effect of holdup ( $\Phi$ ) on static pressure recovery coefficient on outer wall (a) and inner wall (b) for model (1) with $\mathrm{CR}=12.5$ and $\mathrm{Re}=28,500$ (water and stabilized emulsion flows with $0.03 \leq \Phi \leq 0.20$ )
The effects of holdup on the wall-pressure recovery coefficients of model (1) are significant. This is clearly visible for pure water and stable oil-in-water emulsions in Fig. (9) on the outer and inner walls of the model (1). The emulsion holdup values range from 0.03 to 0.20 and with inflow Reynolds number of 28,500. It is clear that the static pressure recovery coefficient of the curved diffusers decreases as the dispersed phase holdup increases, creating a strong adverse pressure gradient inside the curved diffuser. The presence of oil increases the drag of water to move in the curved diffuser and this enhances the energy loss of the curved diffuser.

\section{4-2 Diffuser Performance}

In the present section, attempts have been made to understand the performance of emulsion flow in curved diffuser through the energy-loss characteristics. The diffuser energy-loss coefficient is expressed in terms of inflow Reynolds number. The effects of holdup, area ratio and curvature ratio will be discussed.

The energy loss coefficients of all tested curved diffuser models are presented for pure water as well as stabilized emulsion in Fig. (10).

The emulsion results are considered at different holdup values range from 0.03 to 0.20 .

From the experimental results, the energy-loss coefficient of the curved diffusers is found to be directly proportional to the dispersed phase holdup, which is in agreement with Khalil et al [14].

The energy loss coefficient decreases with Reynolds number because of the reduction of adverse pressure gradient generated in the curved diffuser, while it increases with the emulsion holdup because of the increased drag due to the presence of oil in water which is responsible for the emulsion-viscosity increase.The effects of surfactant presence (stable emulsion) or absence (unstable emulsion) in emulsion on the energy loss in curved diffusers are presented in Figs. (11) and (12).

The study includes the variation of curvature ratio (Fig. 11) and the variation of area ratio (Fig. 12) at a constant holdup of 0.06 . At the same Reynolds number, the energy-loss coefficient of the curved diffuser decreases with increasing either the curvature ratio or area ratio.

The main reason of the curvature effects is the decreasing of the centrifugal force created in the curved diffuser with increasing the curvature ratio, which is responsible for secondary flow generation

For the same radius of curvature, i.e. an equal mainflow passage, the pressure gain from the widest diffuser (the highest area ratio) is higher than the energy lost due intensive secondary flows produced. 


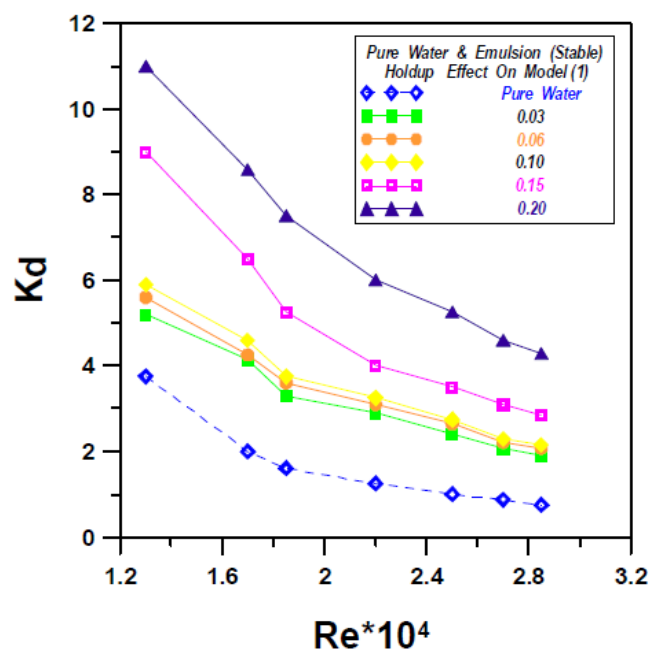

(a) Model (1)

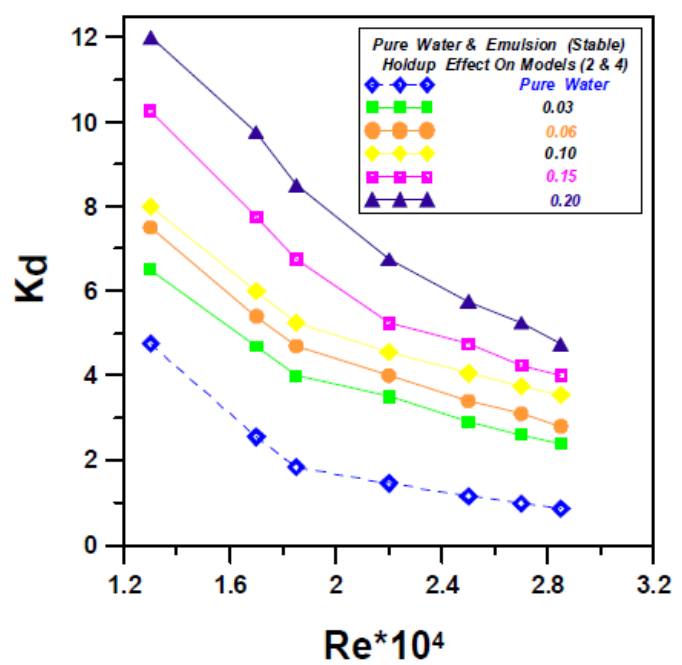

(b) Models (2 \& 4)

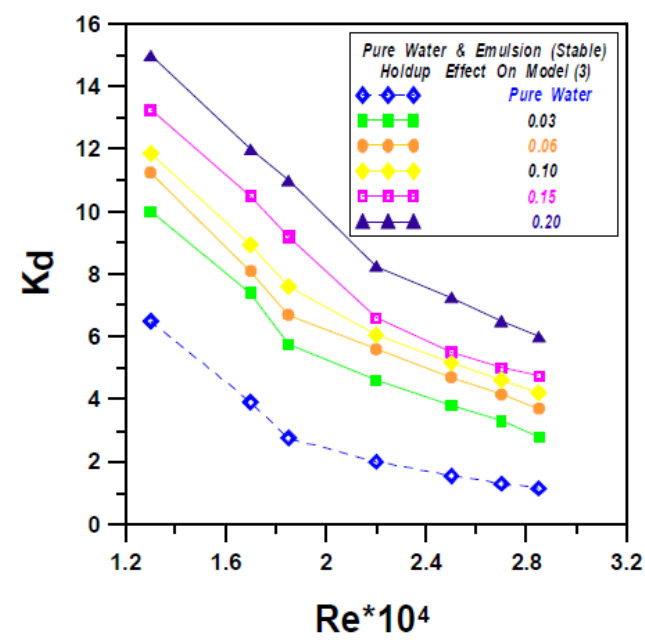

(c) Model (3)

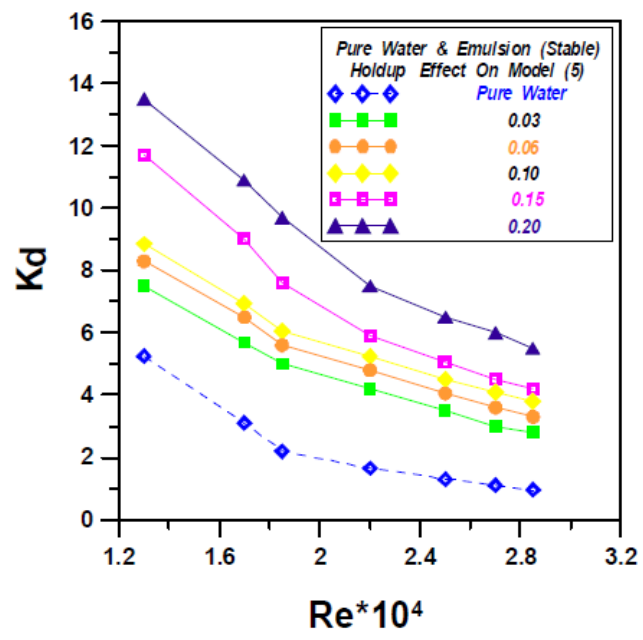

(d) Model (5)

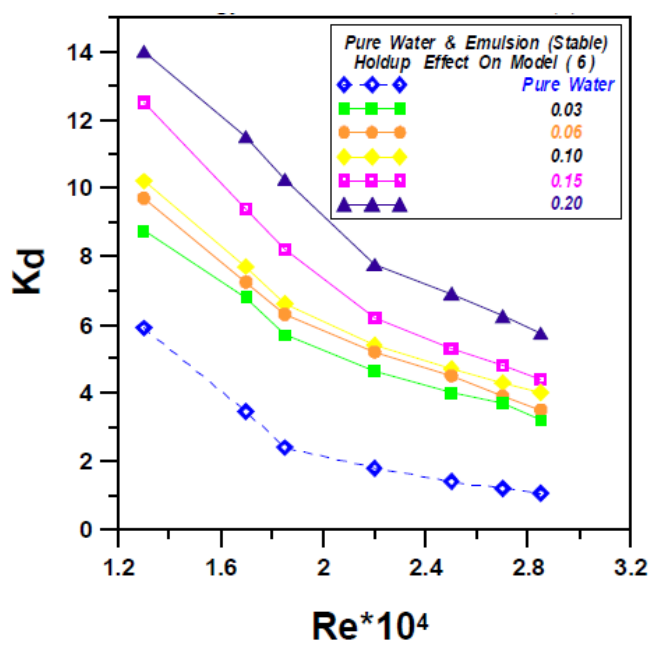

(e) Model (6)

Fig. 10 Diffuser-loss coefficient against Reynolds number (water and stabilized emulsion flows with $0.03 \leq \Phi \leq 0.20$ )

It means that the energy loss decreases with increasing the area ratio. The stable emulsion exhibits greater values of the curved-diffuser energyloss coefficient than the unstable emulsion [ $K_{d}$ (stable-emulsion) $>K_{d}$ (unstable-emulsion)].

This behavior is attributed to the change of turbulence intensity which may be affected by the dispersed phase holdup, area ratio and curvature ratio of the diffuser. 


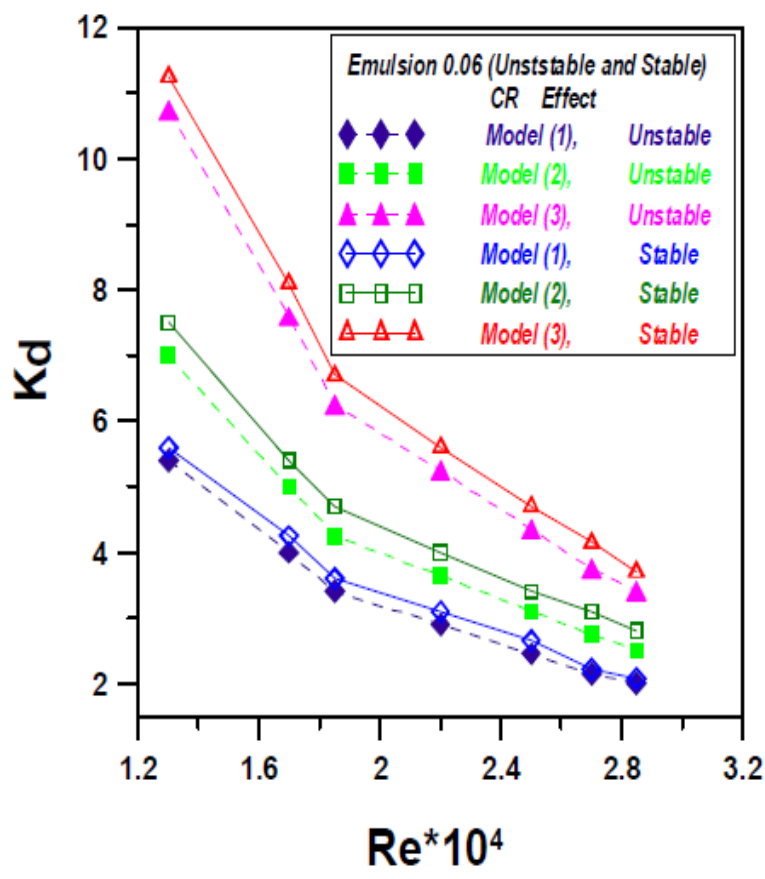

Fig. 11 Effect of curvature ratio on diffuser-loss coefficient (water, stable and unstable emulsion flows with $\Phi=0.06$ )

Figure (13) shows curvature-ratio effects on the energy-loss coefficients of the curved diffuser. The results of water and unstable emulsions flows with two different holdup values ( $\Phi=0.03$ and 0.06 ) are represented in the figure. As discussed previously, as the curvature ratio decreases the energy loss in the curved diffuser increases for the different flows,

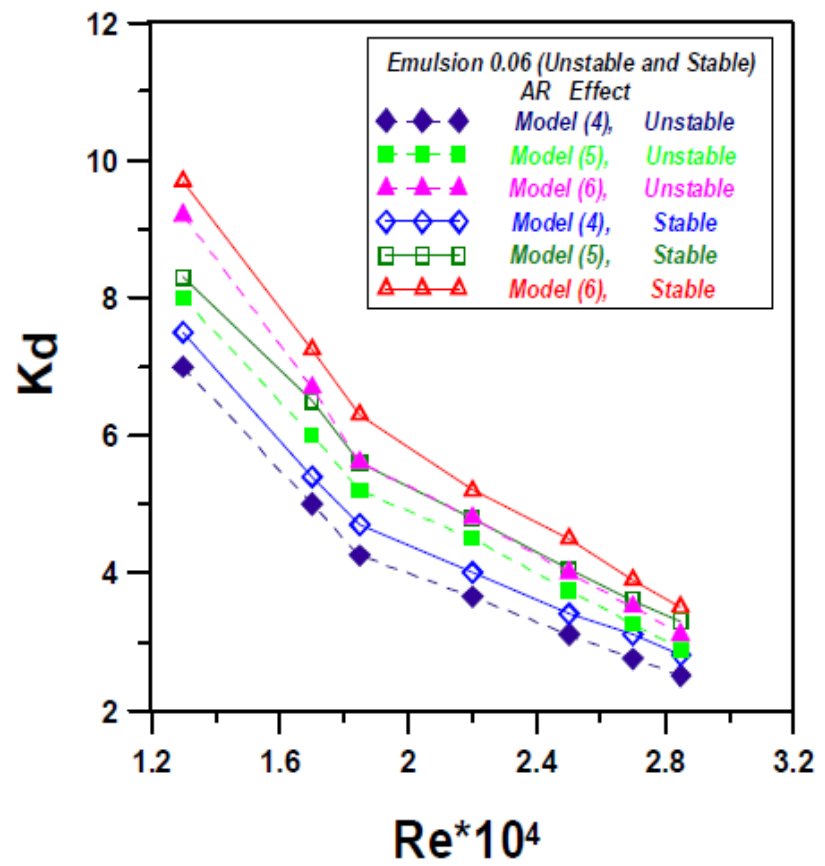

Fig. 12 Effect of area ratio on diffuser-loss coefficient (water, stable and unstable emulsion flows with $\Phi=0.06$ )

because of the generation of strong secondary flows (with small radius of curvature) superimposed on the mean flow.

The curvature ratio effects are again for water and 0.15 holdup stable oil-in-water emulsions in Fig. (14). It is revealed that the pure water flow in curved

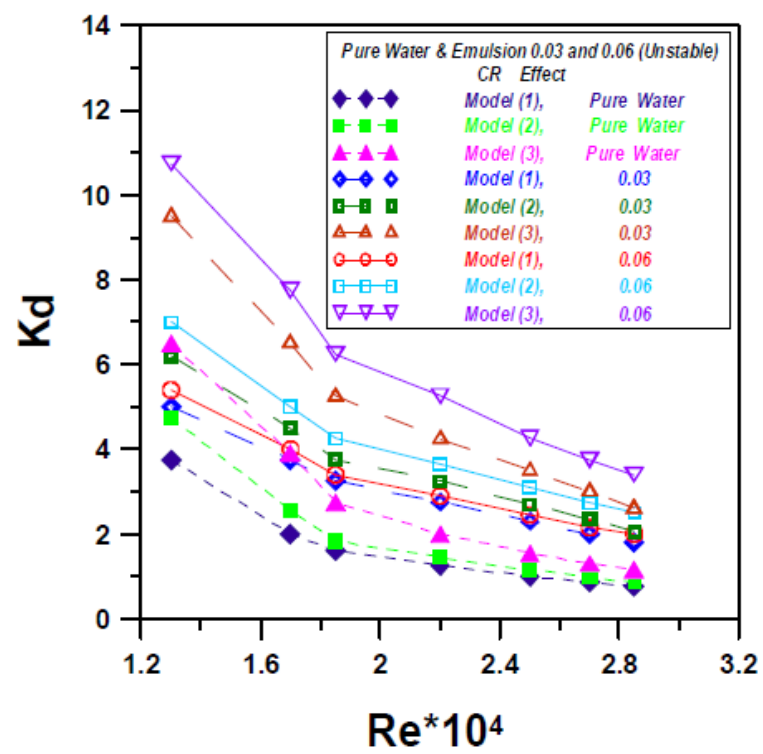

Fig. 13 Effect of curvature ratio and holdup on diffuser-loss coefficient (water and unstable emulsion flows with $\Phi=0.03$ and 0.06 ) 


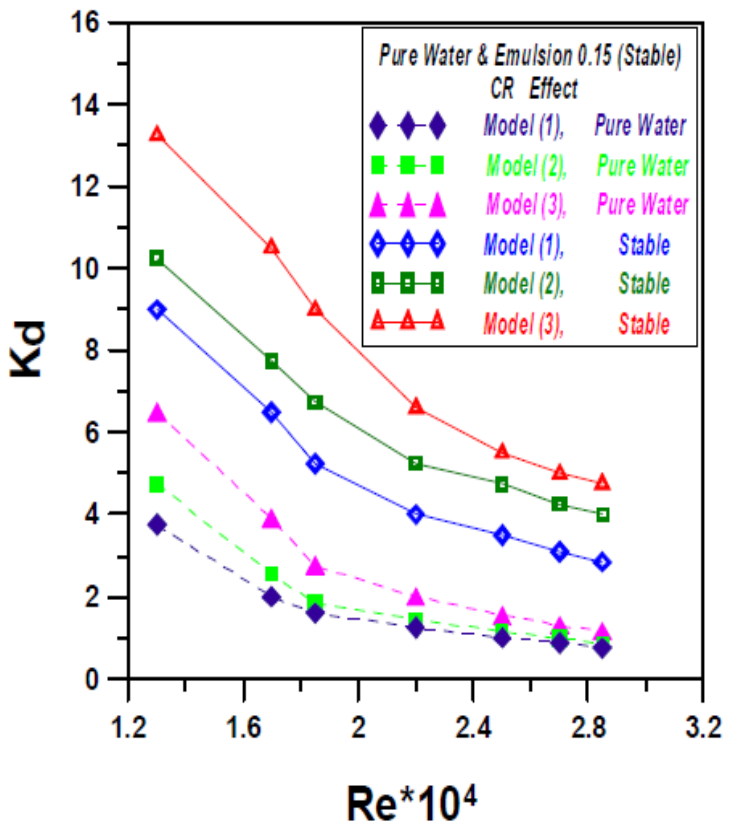

Fig. 14 Effect of curvature ratio on diffuser-loss coefficient (water and stable emulsion flows with $\Phi=0.15$ )

diffusers exhibits the lower values in the energy loss coefficient compared with the stable emulsion flow.

Also the created secondary flows because the strong turn of curved diffuser with small curvature radius clearly increase the curved-diffuser loss coefficient. Generally, the energy-loss coefficient of the curved diffusers for pure water and stable and unstable oilin-water emulsions is found to be inversely proportional to the curvature ratio, area ratio of the curved diffusers and the flow Reynolds number and to approach asymptotic values for high flow Reynolds number due to the intensive turbulence intensity

\section{CONCLUSIONS}

Based on the experimental results, and related discussion the following conclusions can be drawn:

1- The o/w emulsion density increases as the holdup decreases, but the $\mathrm{o} / \mathrm{w}$ emulsion viscosity increases as the holdup increases.

2- For all tested models the pressure recovery coefficient $\left(C_{p}\right)$ decreases through the upstream tangent and the downstream tangent by different values from a curved diffuser model to other. While, it increases through the curved diffuser by different values from a model to other.

3- Under the present range of Reynolds number condition, the curved diffusers pressure recovery coefficients with the stable and unstable o/w emulsions are lower than that for pure water. The unstable o/w emulsion exhibits the highest values compared with stable o/w emulsion.

4- The pressure recovery coefficient decreases as the holdup increases and inflow Reynolds number decreases.

5- Generally, for all tested models the outer wall pressure recovery coefficient is higher than that the inner wall.

6- The curved diffuser energy-loss coefficients with stable and unstable o/w emulsions are higher than that with pure water.

7- The unstable oil-in-water emulsion exhibits lower values in energy-loss coefficients, compared with stable oil-in-water emulsion.

8- The energy-loss coefficient is found to be inversely proportional to the generalized Reynolds number, which is in agreement with that given in literature.

9- For curved diffusers as the area ratio (AR) increases from 1.0 to 2.0, the energy-loss coefficient decreases and as the curvature ratio ( $R_{c} / W$ ) increases from 5 to 12.5 , the energyloss coefficient decreases.

10-Finally, the results and conclusions given in this paper will be useful for those who are working in chemical and petroleum industries.

\section{REFERENCES}

[1] Reneau, L. R., Johnston, J. P. and Kline, S. J. "Performance and Design of Straight, TwoDimensional Diffusers", Journal of Basic Engineering, Transactions of the ASME, Vol. 89, PP. 141-150, (March 1967).

[2] Taylor, J. R., "An Introduction to Error Analysis", University Science Books, Mill Valley, California, (1982).

[3] McMillan, O. J., "Mean-Flow Measurements of the Flow Field Diffusing Bend", NASA Contractor Report 3634, (1982).

[4] Sullerey, R. K., "Performance Comparison of Straight and Curved Diffusers", Defence Science Journal Vol. 33, No 3, July 1983, PP. 195-203, (1983).

[5] Nasr, S., "Hydraulic Resistances" M.Sc. Thesis, Minoufiya University, Egypt (1988).

[6] Pal, R. and Rhodes, E., "Emulsion Flow in Pipelines", Int. J. Multiphase Flow, Vol. 15, No. 6, PP. 1011-1017, (1989).

[7] Pal, R., "Pipeline Flow of Unstable and Surfactant Stabilized Emulsions", AICHE Journal, Vol. 39, No. 11, PP. 1754-1764, (1993). 
[8] Hwang, C-Yi J. and Pal, R., "Flow of TwoPhase Oil/Water Mixture through Sudden Expansions and Contractions", Chemical Engineering Journal, Vol. 68, Issues 2-3, PP. 157-163, (1997).

[9] Turian, R. M., Ma, T.-W., Hsu, F.-L. G., Sung, D.-J. and Plackmann, G. W., "Flow of Concentrated Non-Newtonian Slurries: 2. Friction Losses in Bends, Fittings, Valves, and Venturi Meters", International Journal of Multiphase Flow, Vol. 24, PP. 243-269, (1998).

[10] Pal, R. and Hwang, C-Yi J., "Loss Coefficients for Flow of Surfactant-Stabilised Emulsions through Pipe Components", Trans. IChemE Part A, Vol. 77, PP. 685-691, (1999).

[11] Khalil, M. F., Kassab, S. Z. and Elhalawany, M. M., "Effect Of Surfactant Additives on Energy Loss in Pipe Fittings", 12 th International Mechanical Power Engineering Conference, Mansora, Egypt, (IMPEC 12), PP. F143-F159, (Oct.30-Nov.1, 2001).

[12] Langevin, D., Poteau, S., Henaut, I. and Argillier, J. F., "Crude Oil Emulsion Properties and their Application to Heavy Oil Transportation", Oil \& Gas Science and Technology-Rev. IFP, Vol. 59, No. 5, PP. 511521, (2004).

[13] Ismail, A. S., "Modeling Friction Factor and Drag Reduction of Unstable Water-in-Oil Emulsions in Horizontal Pipes", Al-Azhar University Engineering Journal, Vol. 8, PP. 1024, (2005).

[14] Khalil, M. F., Kassab,S. Z., Ismail, A. S. and Elazab, I. S., "Friction Losses of Oil-in-Water Emulsions Flow Through Pipes", Cairo $10^{\text {th }}$ International Conference on Energy and Enviroment (EE10), Luxor, Egypt, (March 1115, 2007) .

[15] Fester, V., Mbiya, B. and Slatter, P., "Energy Losses of Non-Newtonian Fluids in Sudden Pipe Contractions", Chemical Engineering Journal, Vol. 145, Issue 1, PP. 57-63, (2008).

[16] El-Askary, W.A. and Nasr, M., "Performance of a Bend-Diffuser System: Experimental and Numerical Studies", Journal of Computers and Fluids, Vol. 38(1), PP. 160-170, (2009).

[17] Balakhrisna, T., Ghosh, S. and Das, P.K., "OilWater Flows through Sudden Contraction and Expansion in a Horizontal Pipe- PhaseDistribution and Pressure Drop", International Journal of Multiphase Flow, Vol. 36, Issue 1, PP. 13-24, (2010)

[18] Hammoud, A. H., Yassine, K. C. and Khalil, M. F., "Effect of Oil-in-Water Concentration on the Performance of Centrifugal Pump", Tenth
International Congress of Fluid Dynamics (ICFD 10), Ain Soukhna, Red Sea, Egypt, Paper No. ICFD10-EG-3067, (2010).

[19] Khalil, M. F., Kassab, S. Z., Ismail and A. S., Elazab, I. S., "Energy Losses of Oil-in-Water Emulsions Flow Through Pipe Fittings Using Image Processing", International Review of Mechanical Engineering (I. RE.M.E.), (January, 2011).

\section{NOMENCLATURE}

\section{Symbols}

A Cross-sectional area, $m^{2}$

$B \quad$ Height of the curved diffuser, $m$

$C_{d} \quad$ Discharge coefficient of the orifice flow meter

$C_{p} \quad$ Static pressure recovery coefficient.

$C_{p}(I) \quad$ Ideal Static pressure recovery coefficient

$C_{p_{i}} \quad$ Static pressure recovery coefficient on the inner wall

$C_{p_{o}} \quad$ Static pressure recovery coefficient on the outer wall

$D_{H} \quad$ Hydraulic diameter, $m$

$K_{d} \quad$ Curved diffuser energy loss coefficient

$p \quad$ Pressure, $N / m^{2}$

$\mathrm{P}_{\text {exit }} \quad$ Curved diffuser exit Pressure, $\mathrm{N} / \mathrm{m}^{2}$

$\mathrm{P}_{\text {inlet }} \quad$ Curved diffuser inlet Pressure, $\mathrm{N} / \mathrm{m}^{2}$

Q Fluid Flow Rate, $\mathrm{m}^{3} / \mathrm{s}$

$R_{c} \quad$ Curved diffuser centre-line arc radius, $m$

Re REYNOLDS number

$U_{\text {ref }} \quad$ Mean-streamwise velocity at the upstream reference location

$V_{E} \quad$ Emulsion volume, $\mathrm{m}^{3}$

$V_{\text {exit }} \quad$ Diffuser exit flow velocity, $\mathrm{m} / \mathrm{s}$

$V_{\text {inlet }} \quad$ Diffuser inlet flow velocity, $\mathrm{m} / \mathrm{s}$

$V_{o} \quad$ Oil volume, $\mathrm{m}^{3}$

$V_{w} \quad$ Water volume, $m^{3}$

W Curved diffuser inlet width, $m$ 
K. A. Ibrahim, W. A. El-Askary, E. Wahba1 and Hamdy A. Omara, "Emulsion (Oil-In-Water) Fluid Flow ..."

\begin{tabular}{|c|c|c|c|}
\hline \multirow{3}{*}{$\begin{array}{l}W_{\text {exit }} \\
S\end{array}$} & \multirow{4}{*}{$\begin{array}{l}\text { Curved diffuser exit width, } m \\
\text { dimensional distance along diffuser } \\
\text { centerline, measured from the reference } \\
\text { location, } m\end{array}$} & \multicolumn{2}{|c|}{ Subscripts } \\
\hline & & & Diffuser condition. \\
\hline & & $\mathrm{E}$ & Emulsion \\
\hline \multirow{3}{*}{$S^{*}$} & & exit & Exit \\
\hline & \multirow{2}{*}{$\begin{array}{l}\text { Non-dimensional streamwise distance, } \\
(=S / W)\end{array}$} & I & Ideal \\
\hline & & $\mathrm{i}$ & Inner wall (Convex wall) \\
\hline \multicolumn{2}{|c|}{ Greek symbols } & $o$ & Outer wall (Concave wall) \\
\hline$\Phi$ & Holdup (Ratio of oil volume to emulsion & $\mathrm{O}$ & Oil \\
\hline$\mu$ & Dynamic viscosity, $\mathrm{cP}$ & $\mathrm{p}$ & Pressure recovery \\
\hline$\mu_{E} / \mu_{w}$ & Emulsion/Water viscosity. & ref & Reference value \\
\hline v & Kinematic viscosity, centi Stoke. & $\mathrm{W}$ & Water \\
\hline$\rho_{\mathrm{E}}$ & Mixture density, $\mathrm{Kg} / \mathrm{m}^{3}$ & $x$ & Location value \\
\hline$\rho_{m}$ & Mercury density, $\mathrm{Kg} / \mathrm{m}^{3}$ & $\infty$ & Free-stream value \\
\hline$\rho_{o}$ & Oil density, $\mathrm{Kg} / \mathrm{m}^{3}$ & & \\
\hline$\rho_{w}$ & Water density, $\mathrm{Kg} / \mathrm{m}^{3}$ & & \\
\hline
\end{tabular}

List of abbreviations

AR Area-Ratio of the curved diffuser$$
=A_{\text {exit }} / A_{\text {inlet }}
$$

CR Curvature-Ratio of the curved diffuser$$
=R_{c} / W
$$

$\mathrm{O} / \mathrm{W} \quad$ Oil-in-water emulsion

SDS Sodium dodeycl sulfate

W/O Water-in-oil emulsion

WP Wetted perimeter, m

\section{List of chemical symbols}

$\mathrm{CCL}_{4} \quad$ Carbon Tetra Chloride

$\mathrm{CH}_{3}\left(\mathrm{CH}_{2}\right)_{11} \mathrm{OSO}_{3} \mathrm{Na}$ Sodium Dodeycl Sulfate

Table 1, Geometrical parameters of the curved- diffuser models (dimensions in $\mathrm{cm}$ )

\begin{tabular}{|c|c|c|c|c|c|c|c|c|}
\hline Model & $\boldsymbol{R}_{\mathrm{c}}$ & $\boldsymbol{W}$ & $\boldsymbol{W}_{\text {exit }}$ & $\boldsymbol{B}$ & $\boldsymbol{A}_{\text {inl }}$ & $\boldsymbol{A}_{\text {exit }}$ & $\boldsymbol{C R}$ & $\boldsymbol{A R}$ \\
\hline$(1)$ & 25 & 2 & 4 & 8 & 16 & 32 & 12.5 & 2 \\
\hline$(2) \&(4)$ & 15 & 2 & 4 & 8 & 16 & 32 & 7.5 & 2 \\
\hline$(3)$ & 10 & 2 & 4 & 8 & 16 & 32 & 5 & 2 \\
\hline$(5)$ & 15 & 2 & 3 & 8 & 16 & 24 & 7.5 & 1.5 \\
\hline$(6)$ & 15 & 2 & 2 & 8 & 16 & 16 & 7.5 & 1 \\
\hline
\end{tabular}


K. A. Ibrahim, W. A. El-Askary, E. Wahba1 and Hamdy A. Omara, "Emulsion (Oil-In-Water) Fluid Flow ..."

Table 2, Uncertainty of the energy loss coefficient for curved diffusers with emulsions

\begin{tabular}{|c|c|c|c|c|}
\hline No & Model & $\begin{array}{l}\text { Range of } \\
\mathrm{K}_{\mathrm{d}}\end{array}$ & $\begin{array}{l}\text { Emulsion } \\
\text { type }\end{array}$ & $\begin{array}{l}\text { The percentage } \\
\text { uncertainty } \\
\pm U_{k_{d}} \%\end{array}$ \\
\hline 1 & Model (1) & $1.9 \leq K_{d} \leq 11$ & Stable & $4 \leq \pm U_{k_{d}} \% \leq 4.4$ \\
\hline 2 & Model (1) & $1.8 \leq \mathrm{K}_{\mathrm{d}} \leq 5.4$ & Unstable & $3.1 \leq \pm \mathrm{U}_{\mathrm{k}_{d}} \% \leq 3.5$ \\
\hline 3 & Model (2) & $2.4 \leq K_{d} \leq 12$ & Stable & $3.7 \leq \pm U_{k_{d}} \% \leq 4.2$ \\
\hline 4 & Model (2) & $2.2 \leq K_{d} \leq 7$ & Unstable & $2.9 \leq \pm U_{k_{d}} \% \leq 3.2$ \\
\hline 5 & Model (3) & $2.9 \leq K_{d} \leq 15$ & Stable & $3 \leq \pm U_{k_{d}} \% \leq 3.4$ \\
\hline 6 & Model (3) & $2.6 \leq K_{d} \leq 10.75$ & Unstable & $2.4 \leq \pm U_{k_{d}} \% \leq 2.6$ \\
\hline 7 & Model (5) & $2.7 \leq K_{d} \leq 13.5$ & Stable & $3.4 \leq \pm U_{k_{d}} \% \leq 3.9$ \\
\hline 8 & Model (5) & $2.3 \leq K_{d} \leq 8.0$ & Unstable & $2.7 \leq \pm U_{k_{d}} \% \leq 3$ \\
\hline 9 & Model (6) & $3.2 \leq K_{d} \leq 14$ & Stable & $3.2 \leq \pm U_{k_{d}} \% \leq 3.7$ \\
\hline 10 & Model (6) & $2.4 \leq K_{d} \leq 9.2$ & Unstable & $2.6 \leq \pm U_{k_{d}} \% \leq 2.9$ \\
\hline
\end{tabular}

